\title{
Numerical simulation of bubble generation in a T-junction
}

\author{
S. Arias $^{\mathrm{a}}$, D. Legendre ${ }^{\mathrm{b}}$, R. González-Cinca ${ }^{\mathrm{c}}$ \\ ${ }^{a}$ Escola d'Enginyeria de Telecomunicació i Aeroespacial de Castelldefels, Universitat \\ Politècnica de Catalunya, c/ Esteve Terradas 5, 08860 Castelldefels, Barcelona (Spain) \\ ${ }^{b}$ CNRS, IMFT, Université de Toulouse, INPT, UPS, Institut de Mécanique des Fluides de \\ Toulouse (IMFT), 1 Allée du Professeur Camille Soula, F-31400 Toulouse (France) \\ ${ }^{c}$ Departament de Física Aplicada, Universitat Politècnica de Catalunya, c/ Esteve Terradas 5, \\ 08860 Castelldefels, Barcelona (Spain)
}

\begin{abstract}
We present a numerical study of the formation of mini-bubbles in a 2D T-junction by means of the fluid dynamics numerical code JADIM. Numerical simulations were carried out for different flow conditions, giving rise to results on the behaviour of bubble velocity, void fraction, bubble generation frequency and length. Numerical results are compared with existing experimental data thanks to non dimensional analysis.
\end{abstract}

Keywords: Two-phase flows, Bubble generation, Microgravity, T-junction, Numerical simulation, Volume of Fluid method

\section{Introduction}

In the recent years, a growing interest in the study of gas-liquid flows has arisen as a consequence of their promising technological applications in space $[1,2,3]$. Replacing the widely used single-phase for two-phase systems could lead to an improvement in performance as well as to significant reductions in weight in different fields such as power generation and life support. A good understanding of the behaviour of the gas-liquid interfaces is the cornerstone of these new technologies.

Bubble generation in low gravity environments is a key issue which requires an accurate control. This implies a good knowledge of the interface geometry and the generation of bubbles in a regular way with the smallest possible size dispersion. In this work we focus on the analysis of the formation of a train of 


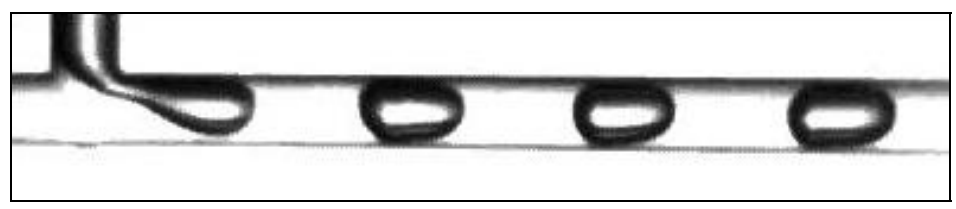

Figure 1: Detail of the bubble generator. Gas is injected from the top and liquid from the left side.

bubbles by means of the cross flow generated in a capillary T-shaped junction $[4,5,6,7]$. In this bubble generator, gas is injected from a capillary into another capillary in a perpendicular direction in which liquid is flowing (see Fig. 1). We consider here the simplest case, in which both capillaries have the same circular cross-section of $1 \mathrm{~mm}$ i.d. Bubbles are generated as a result of the competition between the involved forces, being capillary forces predominant over inertia and buoyancy.

In order to explore the behaviour of the T-junction bubble generator in a wide range of parameters, it is required a reliable numerical code which can complement experimental results. Different computational fluid dynamics methods have been recently used to study the generation of bubbles and droplets in this type or in similar devices. Qian \& Lawal [8] used a commercial CFD package to simulate the bubble formation in the squeezing regime of a T-junction microchannel. Their work was focussed on the study of the effects of pressure, surface tension and shear stress action on the gas thread. Kashid et al. [9] discussed CFD modelling aspects of internal circulations and slug flow generation. The slug flow formation in a $120^{\circ}$ Y-junction was simulated and velocity profiles inside the slug were obtained. More recently, De Menech et al. [10] carried out a numerical investigation by means of a phase-field model of the breakup dynamics of streams of immiscible fluids in a microfluidic T-junction. Three regimes of formation of droplets (squeezing, dripping and jetting) were identified and studied. In spite of the promising results obtained in these recent numerical works, important aspects in the flow characterization such as the bubble generation frequency or the void fraction distribution were not addressed.

The numerical code JADIM developed in the Institut de Mécanique des Fluides de Toulouse (IMFT) has been applied to a variety of fluid dynamics problems $[11,12,13,14,15,16,17,18,19,20]$. The Volume of Fluid (VoF) module of JADIM is able to perform local analyses of deformable two phase interfaces by resolving the Navier-Stokes equations for incompressible fluids in non-stationary problems. An Eulerian description of each phase is applied on a fixed grid and fluids are supposed to be Newtonian. The interface is calculated by means of 
the transport equation of the local volume fraction of one phase, being the surface tension constant and uniform along the interface in the absence of thermal exchange.

In this paper we present a numerical study of the generation of millimetric bubbles in a T-junction by means of JADIM. In Section 2 a dimensional analysis of the bubble generation phenomenon is presented. The numerical code is presented in Section 3 and the modeling of the T-junction is presented in Section 4. Numerical results on the characteristics of the generated flows are presented and compared to existing experimental data in Section 5.

\section{Problem statement}

We consider a 2D T-junction bubble generator. The connection between the two channels as well as the flow directions are shown in Fig. 1. The problem is described using ten independent parameters, namely the gas and liquid densities ( $\rho_{G}$ and $\rho_{L}$, respectively) and viscosities ( $\mu_{G}$ and $\mu_{L}$, respectively), surface tension $\sigma$, capillary diameter $\phi$ (the T-junction being formed by the connection of equal size capillaries), contact angle between the capillaries and the gas-liquid interface $\theta$ (measured on the internal part of the liquid), gravitational constant $g$, and gas and liquid superficial velocities ( $U_{S G}$ and $U_{S L}$, respectively), which are obtained from the air and water volumetric flow rates $\left(Q_{G}\right.$ and $Q_{L}$, respectively):

$$
U_{S G}=\frac{Q_{G}}{A}, \quad U_{S L}=\frac{Q_{L}}{A},
$$

where $A$ is the cross-sectional area of the capillary. Experiments were conducted at a constant temperature around $20^{\circ} \mathrm{C}$ and the system can be assumed adiabatic. According to the Buckingham's $\pi$ theorem, the system can be described by seven dimensionless parameters. The appropriate dimensionless numbers in our study are:

$$
\begin{array}{cc}
\frac{\rho_{L}-\rho_{G}}{\rho_{L}} & B o=\frac{\Delta \rho g \phi^{2}}{\sigma} \\
\operatorname{Re}_{S L}=\frac{\rho_{L} \phi U_{S L}}{\mu_{L}} & \operatorname{Re}_{S G}=\frac{\rho_{G} \phi U_{S G}}{\mu_{G}} \\
W e_{S L}=\frac{\rho_{L} \phi U_{S L}^{2}}{\sigma} & W e_{S G}=\frac{\rho_{L} \phi U_{S G}{ }^{2}}{\sigma}
\end{array}
$$




\begin{tabular}{ccccccc}
$U_{S L}(\mathrm{~m} / \mathrm{s})$ & $U_{S G}(\mathrm{~m} / \mathrm{s})$ & $\operatorname{Re}_{S L}$ & $\operatorname{Re}_{S G}$ & $W e_{S L}$ & $W e_{S G}$ & Regime \\
\hline \hline 0.106 & 0.242 & 106 & 24 & 0.16 & 0.81 & slug \\
\hline 0.106 & 0.344 & 106 & 34 & 0.16 & 1.64 & slug \\
\hline 0.318 & 0.081 & 318 & 8 & 1.40 & 0.09 & bubble-slug transition \\
\hline 0.318 & 0.242 & 318 & 24 & 1.40 & 0.81 & slug \\
\hline 0.318 & 0.337 & 318 & 34 & 1.40 & 1.58 & slug \\
\hline 0.531 & 0.068 & 531 & 7 & 3.92 & 0.06 & bubble \\
\hline 0.531 & 0.236 & 531 & 24 & 3.92 & 0.77 & bubble-slug transition \\
\hline
\end{tabular}

Table 1: Superficial velocities, dimensionless numbers and flow regime observed in each experiment.

Any other dimensionless number should be obtained from the combination of the previous ones. Typically, the Capillary number $\mathrm{Ca}=W e / R e$ is used to compare viscosity and surface tension effects at the interface.

Experiments carried out in $[5,6]$ are used as reference data for the comparison with the simulations reported here. In these experiments, air and water were mixed in a T-junction of two capillaries with $1 \mathrm{~mm}$ of internal diameter. The superficial velocities selected for the comparison with numerical simulations ranged from 0.106 to $0.531 \mathrm{~m} / \mathrm{s}$ for water and from 0.081 to $0.344 \mathrm{~m} / \mathrm{s}$ for air. We considered the following values of the physical properties: $\rho_{L} \simeq 10^{3} \mathrm{~kg} / \mathrm{m}^{3}, \rho_{G} \simeq 1.2 \mathrm{~kg} / \mathrm{m}^{3}$, $\mu_{L} \simeq 10^{-3} \mathrm{~Pa} \cdot \mathrm{s}, \mu_{G} \simeq 10^{-5} \mathrm{~Pa} \cdot \mathrm{s}$ and $\sigma \simeq 0.072 \mathrm{~N} / \mathrm{m}$. According to these values, we obtain $\Delta \rho / \rho \approx 1$ and $B o=0.13$. The values of $U_{S L}, U_{S G}, R e_{S L}, R e_{S G}, W e_{S L}$, $W e_{S G}$, as well as the flow regimes observed in each experiment are shown in Table 1.

In order to carry out the numerical simulations, some changes in the values of two dimensionless parameters $\left(R e_{S L}\right.$ and $\left.R e_{S G}\right)$ had to be considered. In case of taking the same values as in the experiments, the method used for the calculation of the surface tension contribution in the momentum equation, the Continuum Surface Force [21], generates the appearance of spurious currents (see next section for a detailed explanation). These currents induce vortices at the interface without any physical meaning, destabilizing the simulations and strongly distorting the interface [20]. Numerical instabilities produced by the spurious currents depend linearly on the ratio $\sigma / \mu$. For the flow conditions considered here, gas and liquid viscosities had to be increased one order of magnitude in the simulations in order to avoid the spurious currents. Consequently, $\operatorname{Re}_{S L}$ and $\operatorname{Re}_{S G}$ were decreased one order of magnitude for the simulated flows, although both experiments and simulations were carried out at intermediate Reynolds numbers in the 


\begin{tabular}{ccccccc}
$U_{S L}(m / s)$ & $U_{S G}(m / s)$ & $R e_{S L}$ & $\operatorname{Re}_{S G}$ & $W e_{S L}$ & $W e_{S G}$ & Regime \\
\hline \hline 0.106 & 0.242 & 11 & 2 & 0.16 & 0.81 & slug \\
\hline 0.106 & 0.344 & 11 & 3 & 0.16 & 1.64 & slug \\
\hline 0.318 & 0.081 & 32 & 1 & 1.40 & 0.09 & bubble-slug transition \\
\hline 0.318 & 0.242 & 32 & 2 & 1.40 & 0.81 & slug \\
\hline 0.318 & 0.337 & 32 & 3 & 1.40 & 1.58 & slug \\
\hline 0.531 & 0.068 & 53 & 1 & 3.92 & 0.06 & bubble \\
\hline 0.531 & 0.236 & 53 & 2 & 3.92 & 0.77 & bubble-slug transition \\
\hline
\end{tabular}

Table 2: Superficial velocities, dimensionless numbers and regime observed in each numerical simulation.

laminar regime.

We considered in the simulations $g=0$ (thus, $B o=0$ ), while the values of $W e_{S L}$ and $W e_{S G}$ were the same as in the experiments. We also used the same geometry of the capillaries as well as the same gas and liquid superficial velocities as in the experiments. The latter was possible since the width of the capillary in the 2D simulations corresponds to the hydraulic diameter of the experimental T-junction. Under this assumption, the non-dimensional analysis remains valid and the twophase flow behaviour in the simulations is expected to be similar to the observed in the experiments. The superficial velocities, the values of $R e$ and $W e$, as well as the regime observed in each simulation, are shown in Table 2. The corresponding range of the Capillary number is $C a_{S L}=0.015-0.074$.

As regards to the contact angle used in the simulations, its value was chosen in agreement with the observations of the experimental videos (see Section 4.4).

\section{Numerical code}

The implemented VoF method in JADIM consists of an Eulerian description of each phase on a fixed grid, the interface between the two phases being calculated using the transport equation of the local volume fraction of one of the phases. The two fluids are assumed to be Newtonian and incompressible with no phase change. Under isothermal conditions and in the absence of any surfactant the surface tension is constant and uniform at the interface between the two fluids. In such conditions, the velocity field $\mathbf{U}$ and the pressure $P$ satisfy the classical one-fluid formulation of the Navier-Stokes equations:

$$
\nabla \cdot U=0
$$




$$
\frac{\partial U}{\partial t}+U . \nabla U=-\frac{1}{\rho} \nabla P+\frac{1}{\rho} \nabla \cdot \Sigma+g+F_{\sigma}
$$

where $\rho$ and $\mu$ are the density and dynamical viscosity, respectively. $\Sigma$ is the viscous stress tensor, $g$ is the gravity and $F_{\sigma}$ is the capillary contribution:

$$
F_{\sigma}=-\frac{\sigma}{\rho}(\nabla . \mathbf{n}) \mathbf{n} \delta_{I}
$$

where $\mathbf{n}$ denotes by arbitrary choice the unit normal to the interface going out from phase 1 and $\delta_{I}$ is the Dirac distribution associated to the interface. The location of each phase is given by a scalar $C$ (called volume fraction, $\mathrm{VoF}$ function or color function) which obeys the transport equation:

$$
\frac{\partial C}{\partial t}+U \cdot \nabla C=0
$$

This volume fraction is $C=1$ (resp. $C=0$ ) in cells filled with liquid (resp. gas) and $0<C<1$ in cells cut by the interface. Local density and dynamic viscosity are deduced from the value of $C$ by linear interpolation:

$$
\begin{aligned}
& \rho=C \rho_{L}+(1-C) \rho_{G} \\
& \mu=C \mu_{L}+(1-C) \mu_{G}
\end{aligned}
$$

Compared to the classical VoF or Level Set methods [22, 23, 24], the specific aspect of our approach concerns the technique used to control the stiffness of the interface. In our approach no interface reconstruction or redistancing algorithm are introduced. Interface location and stiffness are both controlled by an accurate transport algorithm based on FCT (Flux-Corrected-Transport) schemes [25]. This method leads to an interface thickness of about three grid cells by the implementation of a specific procedure for the velocity used to transport $C$ in a flow region of strong strain and shear [18].

The numerical description of the surface tension is one of the crucial points for the study of systems where capillary effects control the interface shape. This interfacial force is solved using the classical CSF (Continuum Surface Force) model [21] and is distributed over grid points neighboring the interface:

$$
F_{\sigma}=-\frac{\sigma}{\rho} \nabla \cdot\left(\frac{\nabla C}{\|\nabla C\|}\right) \nabla C
$$

The volumetric expression of the capillary term is composed of two terms: one representing the curvature, $H=\nabla \cdot(\nabla C /\|\nabla C\|)$, and the other representing the 
location/orientation, $\nabla C$, of the capillary forcing. A classical problem with this formulation is the generation of spurious currents $[26,27]$ due to a sharp variation of $C$ throughout the transition region between the two phases. In order to decrease the intensity of spurious currents, a classical solution introduced by Brackbill et al. [21] consists of the calculation of the surface curvature from a smoothed density gradient while the discretization of the delta function uses a non-smoothed density. The spurious currents in our code were characterized by Dupont \& Legendre [20] and their maximum magnitude was found to evolve as $\approx 0.004 \sigma / \mu$, in agreement with other codes using the Brackbill formulation.

The volume fraction $C$ and the pressure $P$ are volume-centered and the velocity components are face-centered (see figure 2). Time advancement is achieved through a third-order Runge-Kutta method for viscous stresses. Incompressibility is satisfied at the end of each time step through a projection method. The overall algorithm is second-order accurate in both time and space. A detailed description is given in $[18,20]$. The stability of the numerical simulations is ensured by taking the minimum time step that locally satisfies both the CFL criterion $(=\sqrt{3})$ and the capillary criterion given by

$$
\Delta t_{\sigma}=\sqrt{\frac{\left(\rho_{L}+\rho_{G}\right) \Delta x^{3}}{8 \sigma}}
$$

For the simulations reported here, the time step imposed by $\Delta t_{\sigma}$ is the most restrictive and is about 3 orders of magnitude smaller than the one imposed by the CFL criterion.

The Volume of Fluid (VoF) module of JADIM has been previously validated and used for studies dealing with bubble dynamics in unbounded situations [17, 18], droplets in rectangular mini-channels [19] and sliding drops on inclined walls and in mini-channels [20]. The code is used in this study to simulate the generation of mini-bubbles in a T-junction. 


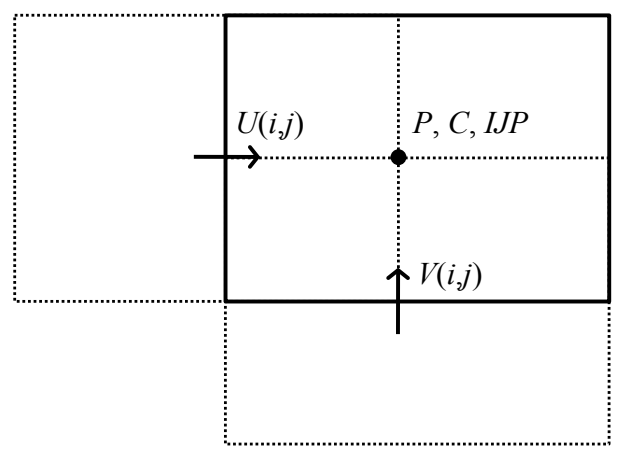

Figure 2: Staggered mesh variable locations and corresponding control volume.

\section{Modelling of the T-junction}

\subsection{Obstacle management}

The T-junction geometry and the associated boundary conditions are managed by the introduction of a Boolean variable, namely IJP. In practice, all the variables are defined from $i=1$ to $N x$ and $j=1$ to $N y$ in the $x$-and $y$-direction, respectively. The cells $(i, j)$ containing the fluid are defined by $\operatorname{IJP}(i, j)=1$, while the value $\operatorname{IJP}(i, j)=0$ is used for the cells without fluid (see figure 3 ). Note that this general formulation makes also possible the treatment of obstacles located inside the fluid domain.

Hence, the calculation of the transport of the $\operatorname{VoF}$ function $C(i, j)$ is done if $I J P(i, j)=1$, and tests using $I J P$ allow to detect the boundaries of the fluid domain. Considering the control volume of the the $\operatorname{VoF}$ function $C(i, j)$, boundary conditions are imposed on the west face if:

$$
\operatorname{IJP}(i, j)=1 \text { and } \operatorname{IJP}(i-1, j)=0,
$$

on the east face if

$$
I J P(i, j)=1 \text { and } I J P(i+1, j)=0,
$$

on the south face if

$$
\operatorname{IJP}(i, j)=1 \text { and } I J P(i, j-1)=0,
$$


and on the north face if

$$
I J P(i, j)=1 \text { and } I J P(i, j+1)=0
$$

The momentum balance is calculated for the components of the velocity located inside the fluid domain and their calculation only involves variables located in the fluid domain or located on the boundaries. Due to the staggered mesh and the location of $U(i, j)$ and $V(i, j)$ (see figure 2), the momentum balance is calculated for the velocity component $U(i, j)$ if:

$$
\operatorname{IJP}(i, j)=1 \text { and } \operatorname{IJP}(i-1, j)=1
$$

and for the component $V(i, j)$ if

$$
\operatorname{IJP}(i, j)=1 \text { and } I J P(i, j-1)=1
$$

Similar tests as (10-13) are done in order to detect the boundaries.

Concerning the Poisson equation, all the nodes (fluid and not fluid) are considered for the resolution. The pressure nodes inside the fluid domain are not connected to the pressure nodes outside the fluid domain since the boundary condition on the wall for the auxiliary potential is:

$$
\frac{\partial \Phi}{\partial n}=0
$$

For the pressure nodes located outside the fluid domain, all the coefficients of the matrix are 0 on the corresponding line except the diagonal term equal to 1 . Thanks to the finite volume formulation and the staggered mesh, the calculation of the source term of the Poisson equation (divergence of the predictor velocity) only involves velocities in the fluid domain or normal to the boundaries (see figure 3 ). For the pressure nodes outside the fluid domain the source term is imposed to 0 .

\subsection{Mesh}

Simulations were performed in a 2D domain with a regular mesh, referred as $M_{1}$. The overall dimensions of $M_{1}$ were $10 \mathrm{~mm} \times 2 \mathrm{~mm}$ (see Fig. 5). The gas and liquid capillary width was $1 \mathrm{~mm} . M_{1}$ contained 600x180 cells, and a vertical diminishing was applied in order to refine it at the T-junction area (see details in Fig. 4(a-c)). The normalized size by the capillary diameter of the largest and smallest cells was $1.67 \cdot 10^{-2} \times 2.45 \cdot 10^{-2}$ and $1.67 \cdot 10^{-2} \times 10^{-2}$, respectively. A 


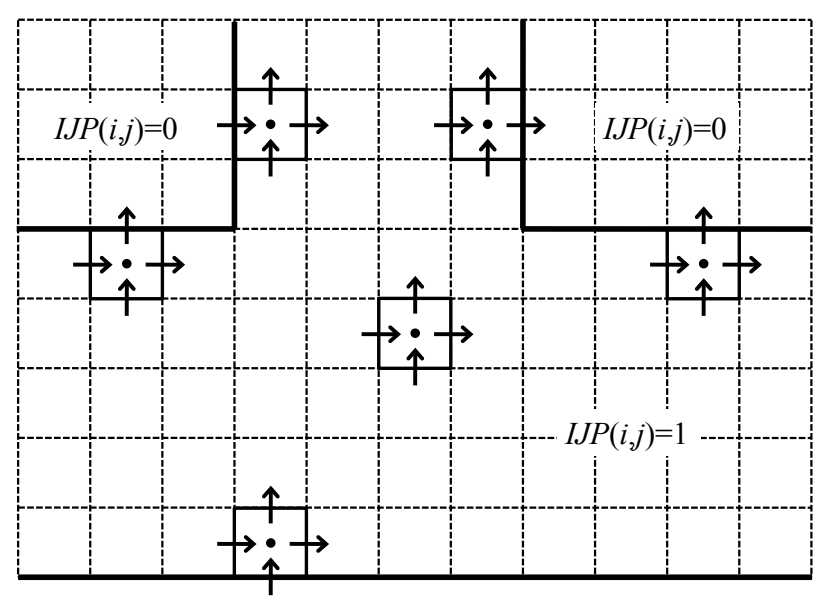

Figure 3: Variable locations in the fluid domain

less refined mesh (referred as $M_{2}$ ), with $300 \times 90$ cells, as well as a more refined mesh with $800 \times 240$ cells (referred as $M_{0}$ ) were also tested. Bubbles with similar regularity and shape were obtained by means of the three tested meshes. In order to test the grid convergence, relative errors were calculated from the following expression:

$$
\varepsilon_{p}=\frac{\left\|p_{i}-p_{0}\right\|}{p_{0}}
$$

where $\varepsilon_{p}$ corresponds to the relative error of a given parameter $p_{i}$ obtained with the mesh $M_{i}(i=1,2)$ with respect to the value $p_{0}$ obtained by the more refined mesh $M_{0}$. Table 3 shows these relative errors for meshes $M_{1}$ and $M_{2}, p_{i}$ being the bubble velocity $U_{G}$, the generation frequency $f$, as well as the bubble and unit cell lengths ( $L_{B}$ and $L_{U C}$, respectively). Tests were carried out with $U_{S L}=0.318 \mathrm{~m} / \mathrm{s}$ and $U_{S G}=0.242 \mathrm{~m} / \mathrm{s}$. A detailed analysis on the behaviour of $U_{G}, f, L_{B}$ and $L_{U C}$ is presented in Section 5. As expected, relative errors were smaller in mesh $M_{1}$ than in $M_{2}$. It is clear from the table that the overall algorithm for the calculation of the bubble characteristics is first-order accurate in space, which is attributed to the numerical squeezing procedure discussed in Section 4.5. The difference between $M_{1}$ and $M_{0}$ being less than $1 \%$, the mesh $M_{1}$ was used for the simulations reported in this work.

Three different time steps $\Delta t_{0}=5 \cdot 10^{-7}, \Delta t_{1}=10^{-6}$, and $\Delta t_{2}=1.31 \cdot 10^{-6}$ 


\begin{tabular}{lcc} 
& $M_{1}$ & $M_{2}$ \\
\hline \hline$\varepsilon_{U_{G}} \cdot 10^{-3}$ & 8.8 & 15.6 \\
$\varepsilon_{f} \cdot 10^{-3}$ & 2.4 & 5.0 \\
$\varepsilon_{L_{B}} \cdot 10^{-4}$ & 1.3 & 3.5 \\
$\varepsilon_{L_{U C}} \cdot 10^{-3}$ & 6.1 & 12.2 \\
\hline
\end{tabular}

Table 3: Bubble velocity, generation frequency, and bubble and unit cell lengths relative errors computed with Eq. 17 for meshes $M_{1}$ and $M_{2}$. Results from $M_{0}$ are used as a reference. Simulations were carried out with $U_{S L}=0.318 \mathrm{~m} / \mathrm{s}$ and $U_{S G}=0.242 \mathrm{~m} / \mathrm{s}$.

\begin{tabular}{lcc} 
& $\Delta t_{1}$ & $\Delta t_{2}$ \\
\hline \hline$\varepsilon_{U_{G}} \cdot 10^{-3}$ & 2.1 & 6.3 \\
$\varepsilon_{f} \cdot 10^{-3}$ & 1.2 & 3.7 \\
$\varepsilon_{L_{B}} \cdot 10^{-2}$ & 1.1 & 2.5 \\
$\varepsilon_{L_{U C}} \cdot 10^{-3}$ & 9.1 & 19.3 \\
\hline
\end{tabular}

Table 4: Bubble velocity, generation frequency, and bubble and unit cell length relative errors computed with Eq. 17 for time steps $\Delta t_{1}$ and $\Delta t_{2}$. Results from $\Delta t_{0}$ are used as a reference. Simulations were carried out with $U_{S L}=0.318 \mathrm{~m} / \mathrm{s}$ and $U_{S G}=0.242 \mathrm{~m} / \mathrm{s}$.

were tested in oder to analyze the time convergence of the simulations. Tests were performed with $U_{S L}=0.318 \mathrm{~m} / \mathrm{s}$ and $U_{S G}=0.242 \mathrm{~m} / \mathrm{s}$. Table 4 shows the relative errors computed for $\Delta t_{1}$ and $\Delta t_{2}$ by means of Eq. 17, in which the value of $p_{i}$ was obtained with a time step $\Delta t_{i}(i=1,2)$. The results obtained with $\Delta t_{0}$ were taken into account in Eq. 17 as the reference values. The relative errors are found to decrease when decreasing the time step, showing the convergence. The corresponding accuracy for the bubble characteristics is found to be first-order in time due to the numerical squeezing procedure. Considering the differences shown in Table 4, the simulations presented in Section 5 were performed with a time step $\Delta t=\Delta t_{2}=1.31 \cdot 10^{-6}$.

\subsection{Contact angle modeling}

A constant contact angle associated to a non slip condition was imposed at the contact point between the gas-liquid interface and the walls defining the Tjunction. The corresponding numerical procedure is described in detail in Dupont \& Legendre [20]. The value of the contact angle is necessary for the calculation of the capillary term contribution given by Eq. 9 for cells in contact with the wall. The numerical scheme consists in expressing the value of the gradient $\nabla C$ of the 


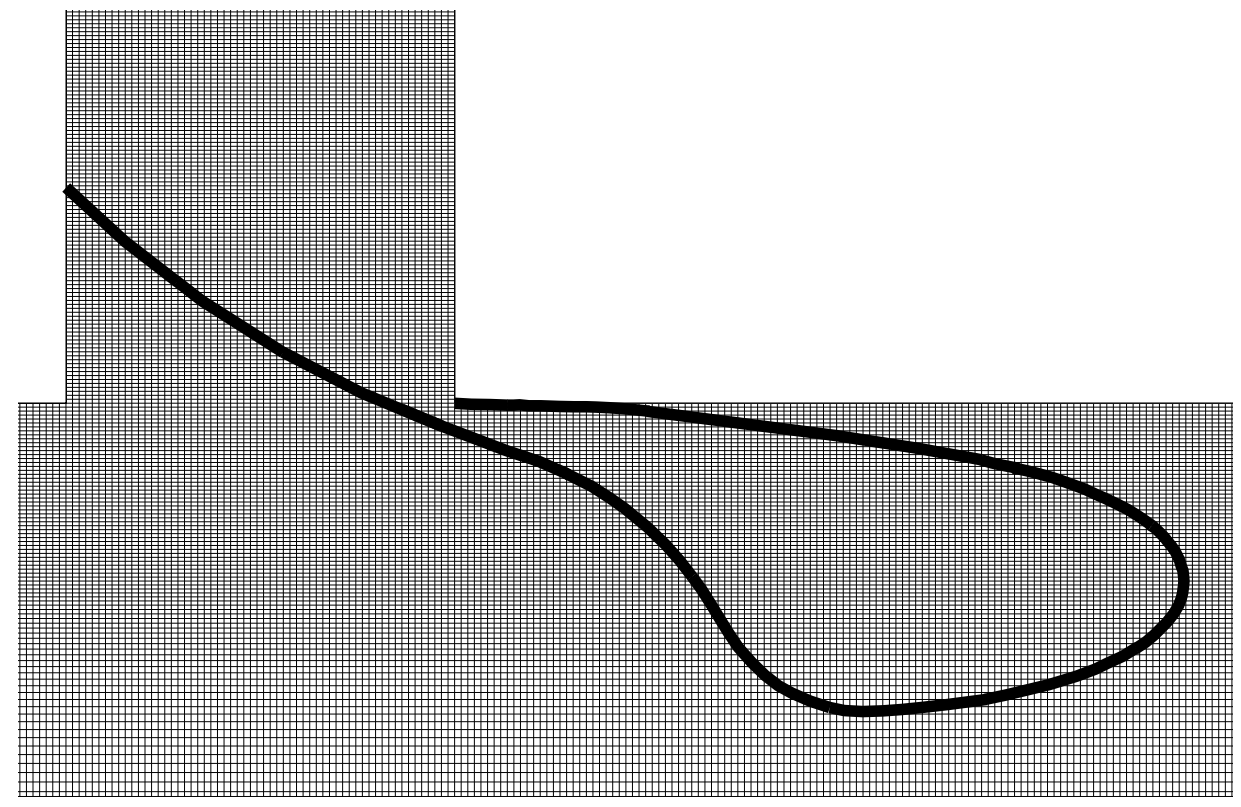

(a)

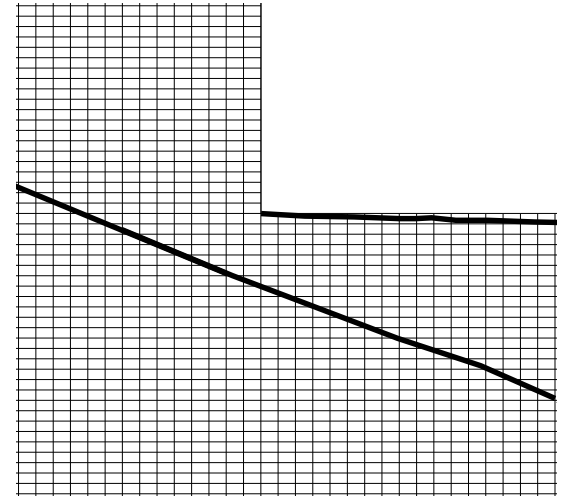

(b)

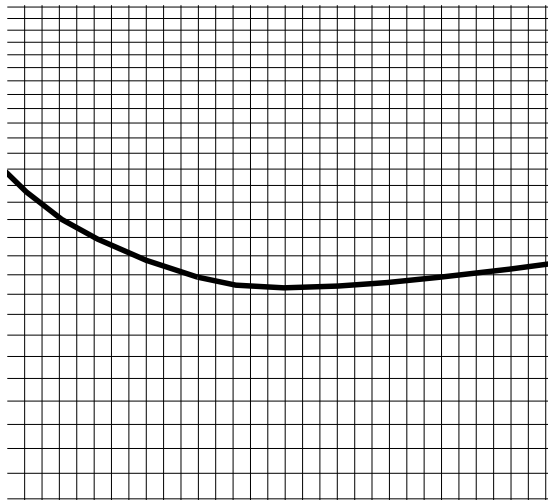

(c)

Figure 4: Mesh used in the simulations. Details of the mesh at $(a)$ the T-junction zone, $(b)$ the gas thread formation zone, and $(c)$ zone at the bottom of the bubble, where the vertical diminishing can be observed. The boundary of the bubble is also plotted as a guideline to the eyes. It does not correspond to the actual thickness of the interface (about 3 grid cells).

volume fraction as a function of the contact angle $\theta$ by means of the relation $\mathbf{n}=\nabla C /\|\nabla C\|=\sin \theta \mathbf{n}_{\|}+\cos \theta \mathbf{n}_{\perp}$, where $\mathbf{n}$ is the normal to the interface and $\mathbf{n}_{\|}$ and $\mathbf{n}_{\perp}$ are the unit vectors in the directions parallel and perpendicular to the wall, 


\begin{tabular}{lc} 
Wall & Boundary conditions \\
\hline \hline 1 & $\theta=25^{\circ}$ \\
\hline 2 & Gas inlet: imposed USG; hydrophobic: $\theta=180^{\circ}$ \\
\hline 3 & $\theta=45^{\circ}$ \\
\hline 4 & Hydrophilic $\theta=0^{\circ}$ and wettability \\
\hline 5 & Outlet \\
\hline 7 & Hydrophilic $\theta=0^{\circ}$ and wettability \\
\hline 8 & Liquid inlet: imposed $U_{S L}$ \\
\hline
\end{tabular}

Table 5: Boundary conditions imposed on the system.

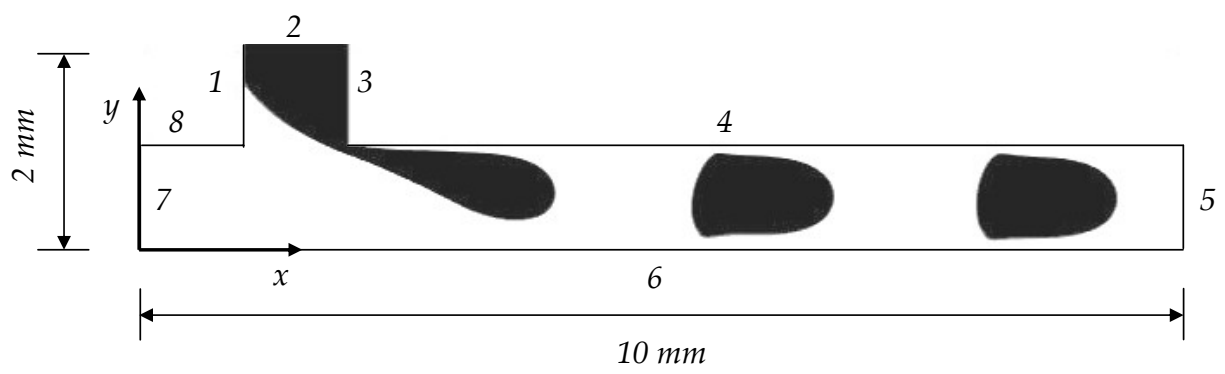

Figure 5: Boundaries, overall dimensions and coordinate axis of the system.

respectively. In order to simplify the calculation of $\nabla C$ at the wall involved in the determination of the capillary force given by Eq. 9, a ghost value for $C$ at the wall is introduced. A validation of the procedure for both constant and dynamic angles has been recently presented by Dupont \& Legendre [20].

\subsection{Boundary conditions}

Results were found to be very sensitive to the conditions imposed on the boundaries of the computational domain. These conditions are summarized in Table 5 for each boundary defined in Fig. 5.

The successful generation of bubbles relies especially on a good selection of boundary conditions associated to wall 1 , since they determine the curvature of the rear interface and therefore the bubble shape. The aim of this work is to provide numerical simulations for the description of the hydrodynamic aspects of bubble generation for given wetting conditions. The study of the wetting effects on the bubble generation is in itself a very interesting subject but it is not addressed here. In order to make possible some relevant comparisons with the experiments, it is 
important to choose an appropriate set of parameters for the wetting conditions. However, the wetting properties of the solid surface used in the experiments are not known. Different values of the contact angles are clearly shown in the experimental images (see figure 1). In order to overcome this point, we have deduced the values of the contact angles from the experimental images in one case, and the same wetting conditions have been used for all the simulations reported in this paper.

Considering wall 1, the contact angle determines the attachment of the gas to the vertical capillary (see Fig. 6). Fixing a $0^{\circ}$ contact angle resulted in a too restrictive condition that forced the gas to remain attached at the upper side of the wall and the rear interface of the bubble to tilt back excessively in comparison with experiments. On the other hand, imposing a $90^{\circ}$ contact angle quickly stabilized the gas at the lower corner of the wall resulting in unrealistic interfaces. We used images obtained from the experiments in order to determine the appropriate contact angle to impose on wall 1 (see Fig. 7). The capillary curvature and the insufficient illumination were disadvantages for the quality of the measurements. In addition, liquid drag and gas fluctuations after the breakup of the bubble made the contact angle to be dynamic. However, selecting a fixed value $\theta=25^{\circ}$, we obtained a generation of bubbles whose interface shape at wall 1 was in good agreement with experimental observations. We also observed that small variations $\left( \pm 15^{\circ}\right)$ around the selected value do not seem to affect noticeably the results. One can observe in Fig. 6 the different interfaces obtained with $\theta=0,25$ and $90^{\circ}$, where the case with an intermediate value shows a shape closer to the experiments. Note that during the bubble generation the contact line in wall 1 is first observed to move from the liquid to the gas (advancing angle) before it stops and in wall 3 the contact line is observed to be fixed so that both contact angles during bubble generation are somewhere in the hysteresis. A $45^{\circ}$ contact angle was imposed on wall 3 to force the forward inclination of the frontal interface in agreement with the experimental image. Nevertheless, some changes in the contact angle value around $45^{\circ}$ did not show any significant impact on the results.

As regards to walls 4,6 , and 8 , hydrophilic $\left(\theta=0^{\circ}\right)$ and wet boundary conditions were imposed in order to prevent bubbles from attaching to them. Note that the value of the contact angle in these walls have not an effect on the simulations. Walls 2 and 7 were defined as fluid inlets and wall 5 was defined as a fluid outlet. The corresponding gas and liquid superficial velocities were thus imposed on walls 2 and 7. 


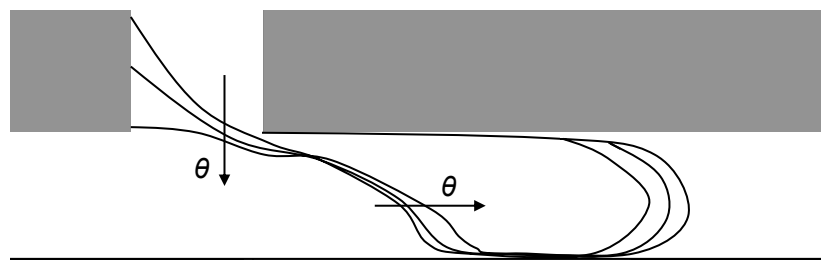

Figure 6: Influence of the contact angle imposed on wall 1 on the interface shape, for $U_{S L}=0.318$ $\mathrm{m} / \mathrm{s}$ and $U_{S G}=0.182 \mathrm{~m} / \mathrm{s}$. Lines correspond to $\theta=0,25$ and $90^{\circ}$.

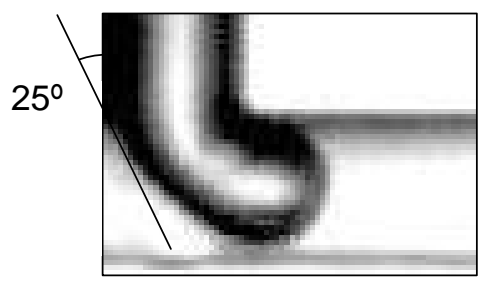

Figure 7: Contact angle at wall 1 measured from experimental images.

\subsection{Gas squeezing}

According to the experimental observations, bubble generation results from the breakup of a gas thread that develops after the T-junction. The explanation for the breakup is supported by different theories. Its cause can be explained by the Plateau-Rayleigh instability [28] or by the effects of the flowing liquid from the tip of the thread to the neck where pinch-off occurs [29]. In 2D, the surface tension has a stabilizing effect and opposes any deformation of the interface tending to create a bubble. This is in agreement with our simulations since no natural pinch-off has been observed for the range of parameters covered by our study. An example is shown in Fig. 8, where a long thread of gas generated after the T junction is clearly observed. It is found to be very stable and remains after the bubble exits the computational domain. In addition, the thread width, $h$, was also found to be grid independent when refining the grid. A comparison between the gas thread generated for the three different meshes considered $\left(M_{0}, M_{1}\right.$ and $\left.M_{2}\right)$ was carried out. To this end, $h$ was measured at $x=2 \mathrm{~mm}$ (see x-axis in Fig. 5), which corresponds to one of the corners where the two capillaries intersect. At this corner, $h$ reaches a minimum in our simulations in agreement with the experiments. Fig. 9 shows the time evolution of $h$ normalized with the capillary diameter for meshes $M_{0}, M_{1}$ and $M_{2}$. The figure clearly confirms the time convergence of the simulations.

In order to be able to generate bubbles in a 2D geometry, an artificial gas 


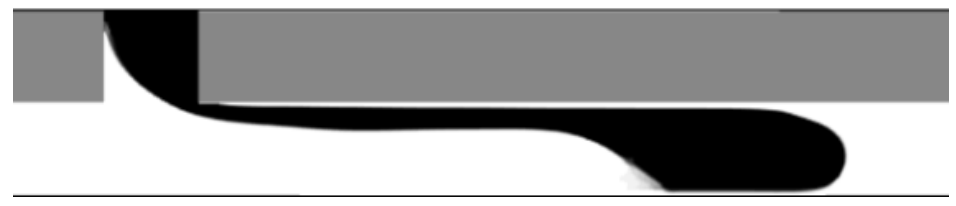

Figure 8: Gas thread generation without squeezing. $U_{S L}=0.318 \mathrm{~m} / \mathrm{s}$ and $U_{S G}=0.182 \mathrm{~m} / \mathrm{s}$.

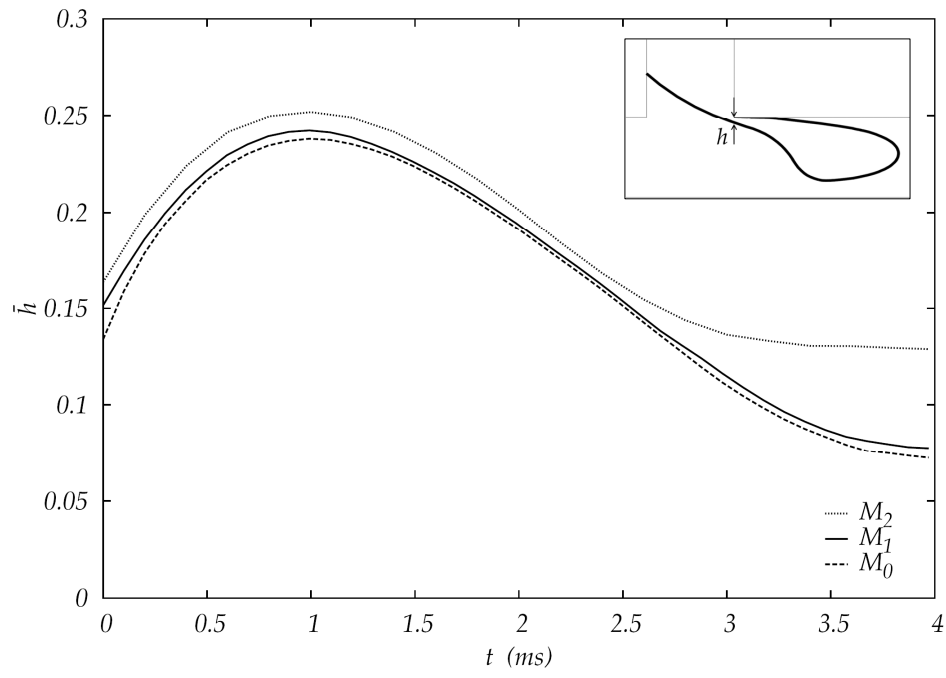

Figure 9: Time evolution of the normalized thread width $\bar{h}$ for meshes $M_{0}, M_{1}$ and $M_{2} . U_{S L}=$ $0.318 \mathrm{~m} / \mathrm{s}$ and $U_{S G}=0.242 \mathrm{~m} / \mathrm{s}$. 
squeezing mechanism was introduced in JADIM. According to the experimental observations used for comparison in the next section (see Figs. 12-14), the gas squeezing is generated where the curvature $H$ of the interface cancels. An inflection point $(H=0)$ is thus present at the interface. Close to this point the gas thread reaches a minimum, making possible the development of the squeezing mechanism. The same precursor behavior is observed in our 2D simulations. Thus, we have implemented the squeezing of the gas thread at the inflection point location $(H=0)$ when $h$, the thread width at the right corner of the T-junction located at $x=2 \mathrm{~mm}$, reaches its minimum stabilized value. The time evolutions of $h$ and the $x$-position of the inflection point $x_{b p}$ are shown in Fig. 10 in order to illustrate the numerical procedure. $h$ and $x_{b p}$ have been normalized by the capillary diameter. Fig. 10 shows a period of bubble generation once the steady state has been reached. The plot starts just after the detachment of the previous bubble. Consequently, $h$ starts from a minimum value corresponding to the beginning of the growth of a new bubble (point (a) in Fig. 10). Then, $h$ increases and reaches a maximum value at point (b). The rear interface of the bubble is rounded during the early stages of the bubble formation and it becomes mainly flattened once $h$ has reached its maximum value. Next, $h$ decreases and the sign of the interface curvature changes (point (c)). At this moment the time evolution of the point inflection location $x_{b p}$ starts. The evolution of $x_{b p}$ shows that the inflection point moves downstream as the gas thread and the bubble grow. When $h$ reaches its minimum at the right corner of the T-junction the squeezing procedure is applied (point (d) in Fig. 10). The artificial gas squeezing mechanism consists in the removal of the gas cells where $H=0$, corresponding to $x=x_{b p}$. For this purpose, the cells $(i, j)$ and $(i+1, j)$ are filled with liquid if $i$ satisfies $x_{C i, j} \leq x_{b p} \leq x_{C i+1, j}$, where $x_{C i, j}$ is the x-coordinate of $C$ in cell $(i, j)$. The total gas volume lost under the application of this artificial squeezing mechanism is about a few grid cells and can be considered negligible compared to the bubble volume. This procedure is in agreement with experimental observations where the 3D instability squeezes the thread at the inflection point. The bubble thread before squeezing in both experiments and simulations is shown in Fig. 11.

After squeezing, the shape of the injected gas jet is naturally adjusted as a result of the action of surface tension, generating the appropriate initial conditions for the following bubble. Although the initial shape of the gas jet in the simulations had a strong influence on the first generated bubble, it does not show any influence on successive bubbles. The second generated bubble had the same size as the following ones, fixing the periodicity of the process. Thus, we can conclude that the initial shape of the gas jet does not play any significant role in the 


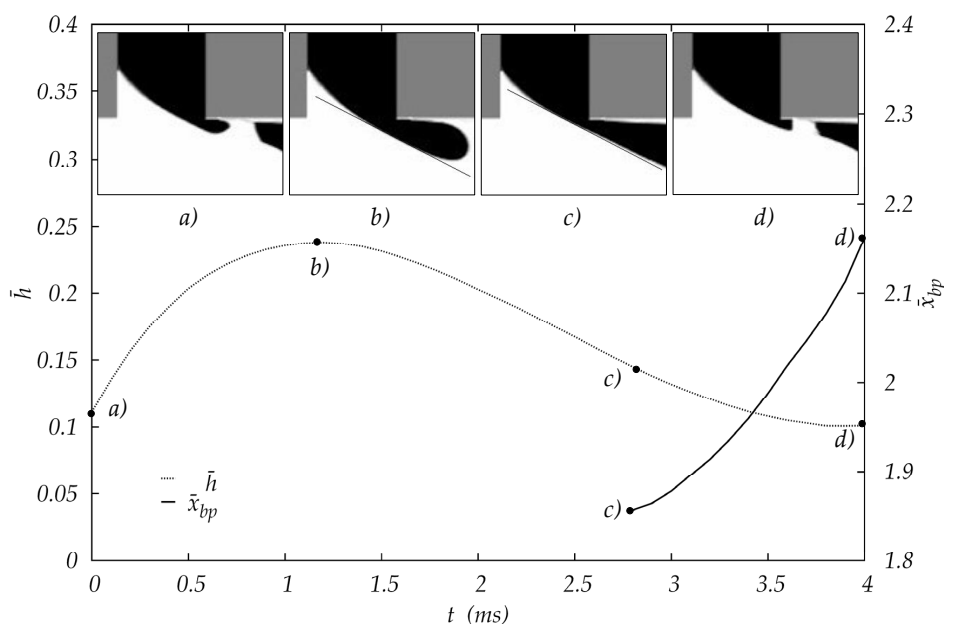

Figure 10: Time evolution of the normalized thread width, $\bar{h}$, and the normalized position of the inflection point, $\bar{x}_{b p}$. Details of the T-junction at $(a)$ detachment of a bubble, $(b)$ maximum $\bar{h}$, (c) appearance of the inflection point, and (d) gas thread breakup. $U_{S L}=0.318 \mathrm{~m} / \mathrm{s}$ and $U_{S G}=$ $0.242 \mathrm{~m} / \mathrm{s}$.

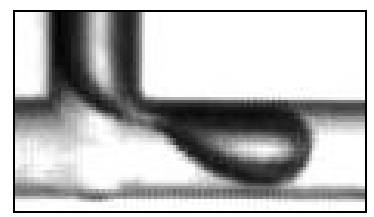

(a)

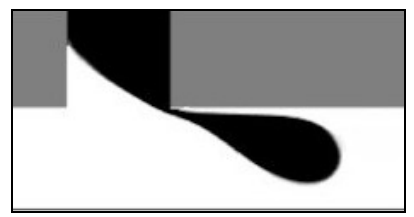

(b)

Figure 11: Comparison of the bubble thread just before being squeezed in $(a)$ experiments and $(b)$ numerical simulations. $U_{S L}=0.318 \mathrm{~m} / \mathrm{s}$ and $U_{S G}=0.081 \mathrm{~m} / \mathrm{s}$. 
generation of a train of bubbles. This turned out to be an essential feature of the numerical simulations, since no theoretical predictions or experimental data on the initial shape were available.

\section{Results and discussion}

A set of seven numerical simulations were performed by means of JADIM. We used the same injection conditions (liquid and gas superficial velocities) as in the experiments. Tables 1 and 2 show the injection conditions as well as the observed flow patterns in the experiments and simulations, respectively. Simulations successfully reproduced different trains of bubbles (see comparison with experiments in Figs. 12-14, in which the process of generation of a single bubble is shown in each case), obtaining a regular periodicity in the bubble generation and regularity in the bubble size, as will be discussed in Sections 5.2 and 5.3, respectively. As can be observed, the time required for the formation of a bubble is shorter in the numerical simulations than in the experiments, which will be discussed in (Section 5.2).

Both bubble and slug flow patterns, defined as proposed by Dukler et al. [30], were observed in the simulations. Under this definition, the transition between bubble and slug regimes is considered to take place when the bubble diameter reaches the value of the capillary diameter.

For a given $U_{S G}$ and smaller values of $U_{S L}$ than those proposed here, a marginal churn flow pattern was also observed in the experiments [6], although it is not considered in this numerical study. Moreover, we did not expect to observe annular or stratified flow patterns due to the small values of $U_{S G}$ and the capillary diameter (smaller than the critical diameter described in Suo \& Griffith [31]), respectively.

Fig. 12 shows a slug flow obtained with $U_{S L}=0.106 \mathrm{~m} / \mathrm{s}$ and $U_{S G}=0.344 \mathrm{~m} / \mathrm{s}$. A slight increasing of the value of $U_{S G}$ would cause the flow to enter into the slug-churn transition region. Figs. 13 and 14 show examples of slug and bubble flow patterns obtained with $U_{S L}=0.318 \mathrm{~m} / \mathrm{s}$ and $U_{S G}=0.242 \mathrm{~m} / \mathrm{s}$, and $U_{S L}=0.531$ $\mathrm{m} / \mathrm{s}$ and $U_{S G}=0.068 \mathrm{~m} / \mathrm{s}$, respectively. Clear similarities in the bubble shape in both patterns between experiments and numerical simulations can be observed. Bullet-shaped bubbles rounded at the front and flattened at the rear were obtained in the slug flow. Bubbles generated in the bubble flow regime were deformed as a consequence of being longitudinally dragged by the continuous phase. Numerical simulations reproduced the experimentally observed fluctuations at the back of the bubbles following the breakup of the gas thread and the subsequent action of surface tension to reduce the interface. 

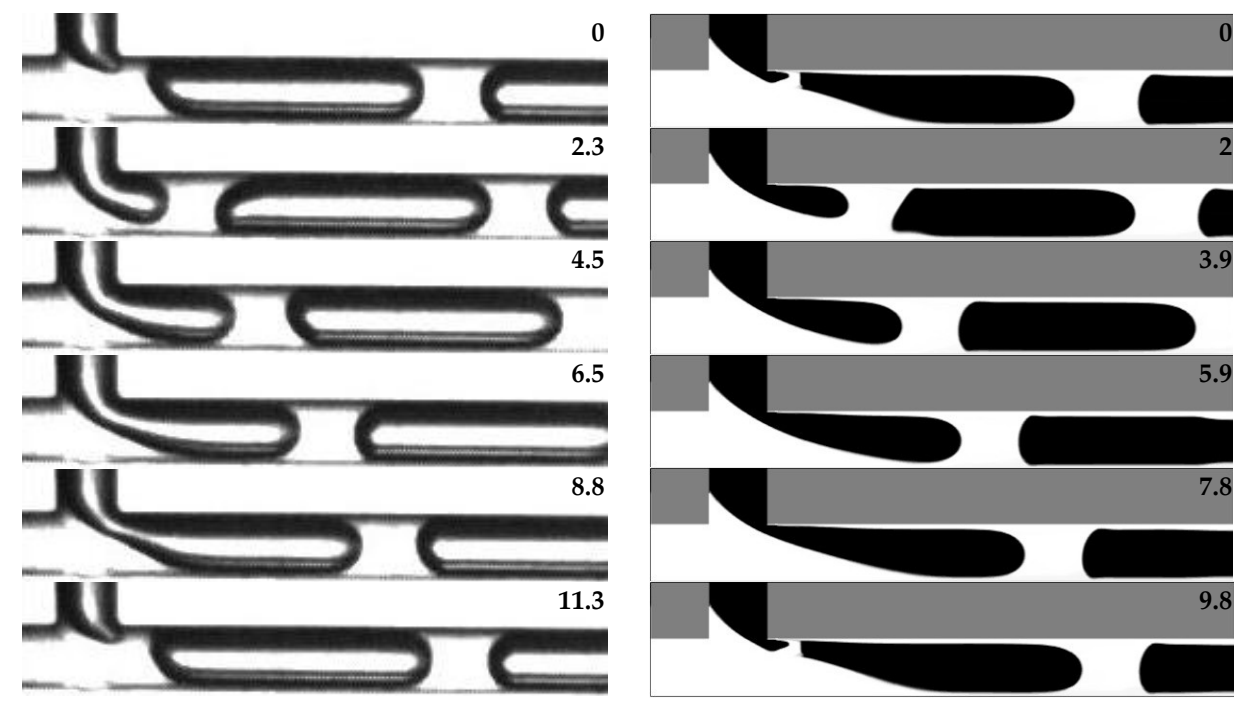

Figure 12: Slug flow close to the slug-churn transition in (left) experiments and (right) numerical simulations. $U_{S L}=0.106 \mathrm{~m} / \mathrm{s}$ and $U_{S G}=0.344 \mathrm{~m} / \mathrm{s}$. Time $(\mathrm{ms})$ is indicated in the upper right corner.
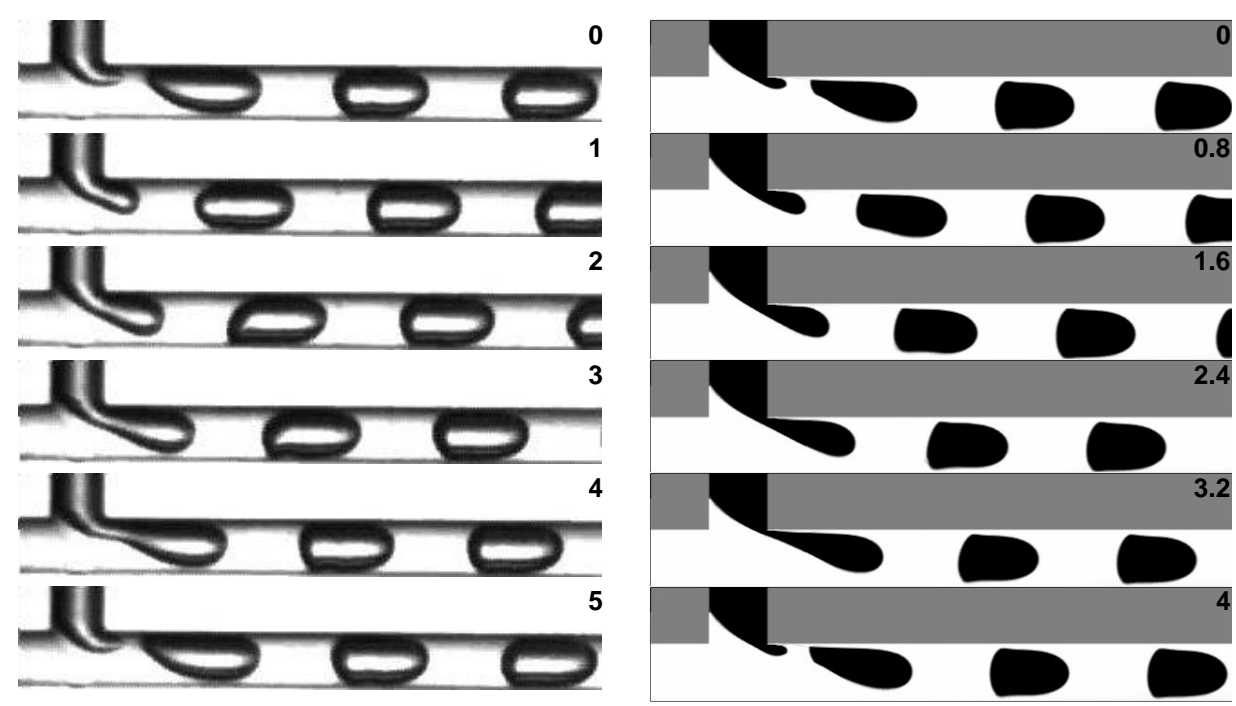

Figure 13: Slug flow in (left) experiments and (right) numerical simulations. $U_{S L}=0.318 \mathrm{~m} / \mathrm{s}$ and $U_{S G}=0.242 \mathrm{~m} / \mathrm{s}$. Time $(\mathrm{ms})$ is indicated in the upper right corner. 

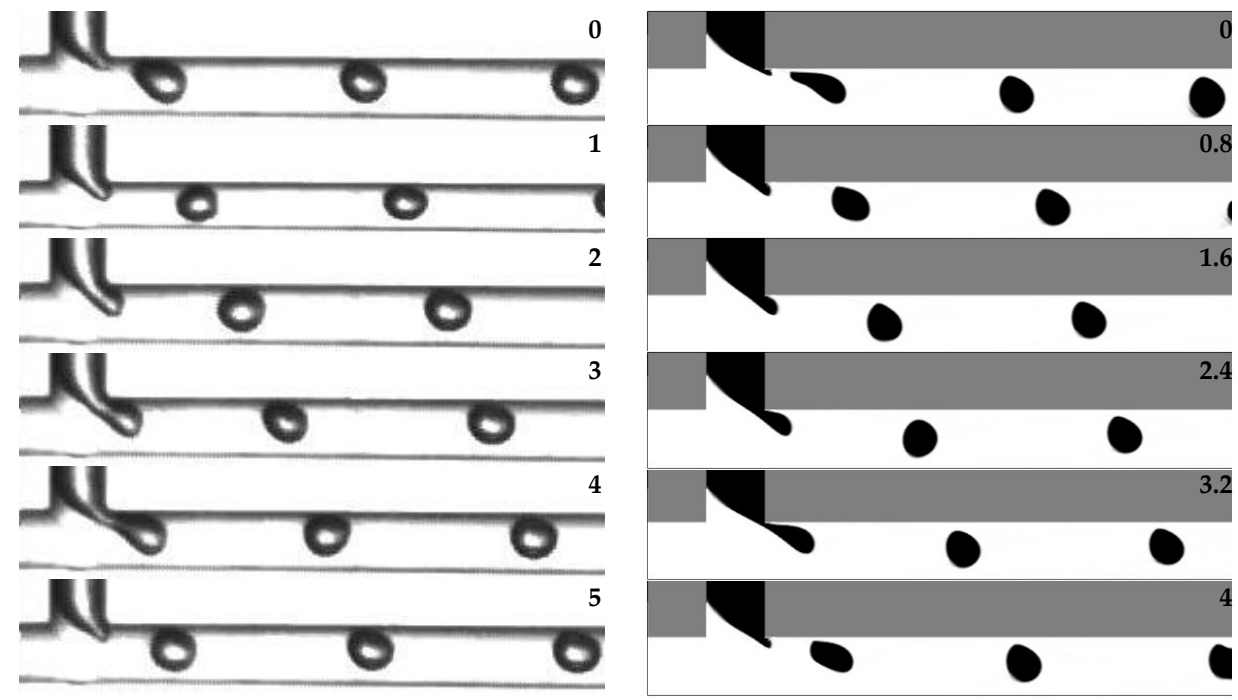

Figure 14: Bubble flow in (left) experiments and (right) numerical simulations. $U_{S L}=0.531 \mathrm{~m} / \mathrm{s}$ and $U_{S G}=0.068 \mathrm{~m} / \mathrm{s}$. Time ( $\mathrm{ms}$ ) is indicated in the upper right corner.

Note that in the simulations the bubbles are moving along the centerline while in the experiments gravitational effects are still acting, which results in the bubble displacement from the capillary centreline.

The flow patterns obtained in the experiments and numerical simulations are compared in Fig. 15 for the same gas and liquid superficial velocities. As can be observed, the same flow regimes were obtained in experiments and numerical simulations when the superficial velocities of the experiments were used as inputs in the simulations (see also Tables 1 and 2).

\subsection{Bubble velocity and void fraction}

The bubble velocity is known to exceed the average speed of the fluid and is usually expressed using the drift-flux relationship, which we express here considering zero-gravity conditions:

$$
U_{G}=C_{0}\left(U_{S L}+U_{S G}\right)
$$

Different values for $C_{0}$ can be found in the literature depending on both the geometry and the effects of inertia, viscosity and surface tension. According to Nicklin et al. [33], the bubble velocity is close to the axis liquid velocity far upstream so that $C_{0}$ is about 2 for laminar pipe flow and about 1.2 for turbulent pipe flow. This is confirmed by the experiments of Colin et al. [34], Bousman et al. [35] where $C_{0}$ 


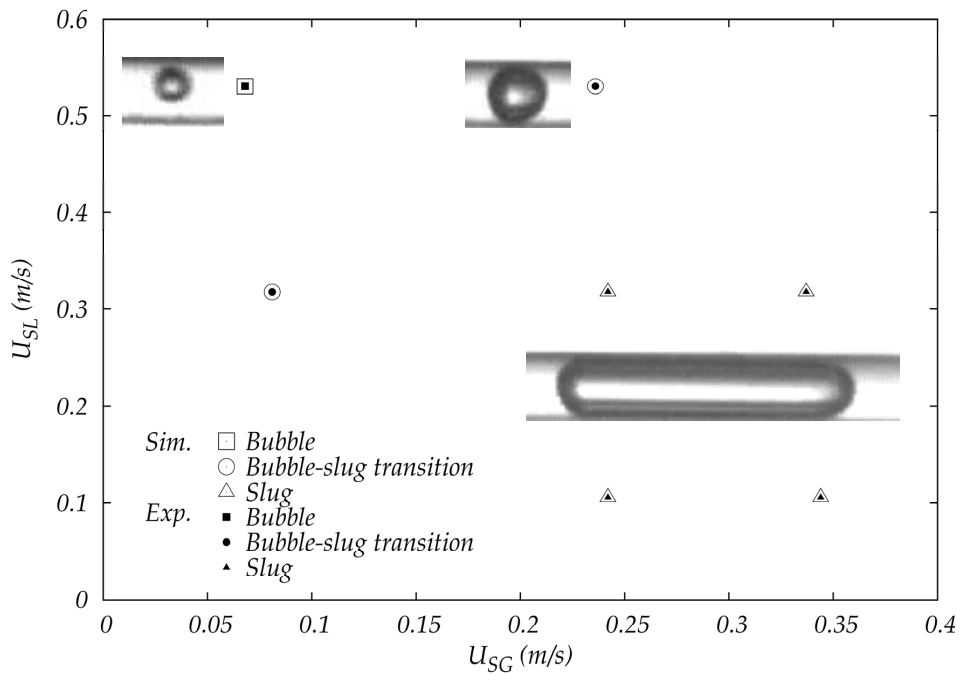

Figure 15: Flow pattern map obtained from experimental and numerical data.

is found to be close to 1.2 for turbulent pipe flow, as well as by the experiments of Taylor [36] at small Reynolds number where a nearly constant value of $C_{0} \sim 2.3$ is observed for Capillary number $C a_{L}=\mu_{L} U_{L} / \sigma$ larger than 1.5. In the limit of small both Capillary and Reynolds numbers, the difference between bubble velocity and liquid velocity is found to evolve as $\mathrm{Ca}^{2 / 3}$ [37] so that $C_{0}$ tends to unity as confirmed by recent experiments [38, 39].

Concerning 2D channel flows, the same trends are expected. For laminar flows, the inviscid numerical solution reveals that $C_{0}=1.4$ [40], and Navier Stokes simulations for laminar flow but in the limit of both large Reynolds and Capillary numbers gives $C_{0}=1.37$ [41]. This is in agreement with the consideration of Nicklin et al. [33] that predicts $C_{0}=1.5$ for the laminar channel flow. The opposite limit for small Reynolds and Capillary numbers is $C_{0}=1$.

We estimated the bubble velocity in experiments and simulations from the measurement of the displacement of the front part of the bubble and the time employed in this displacement.

Fig. 16 shows the bubble velocity as a function of the mixture superficial velocity $U_{T}=U_{S L}+U_{S G}$. A linear behaviour can be observed in both the experiments and the numerical simulations. Fitting the simulation data gives a slope $C_{0}=1.21$, which agrees with the value reported in the literature since $C_{0}$ is expected to range from 1 to 1.4 in $2 \mathrm{D}$. The fitting of the experimental data shown here gives a slope $C_{0}=1.08$ which is also in agreement with previous results in pipes. 


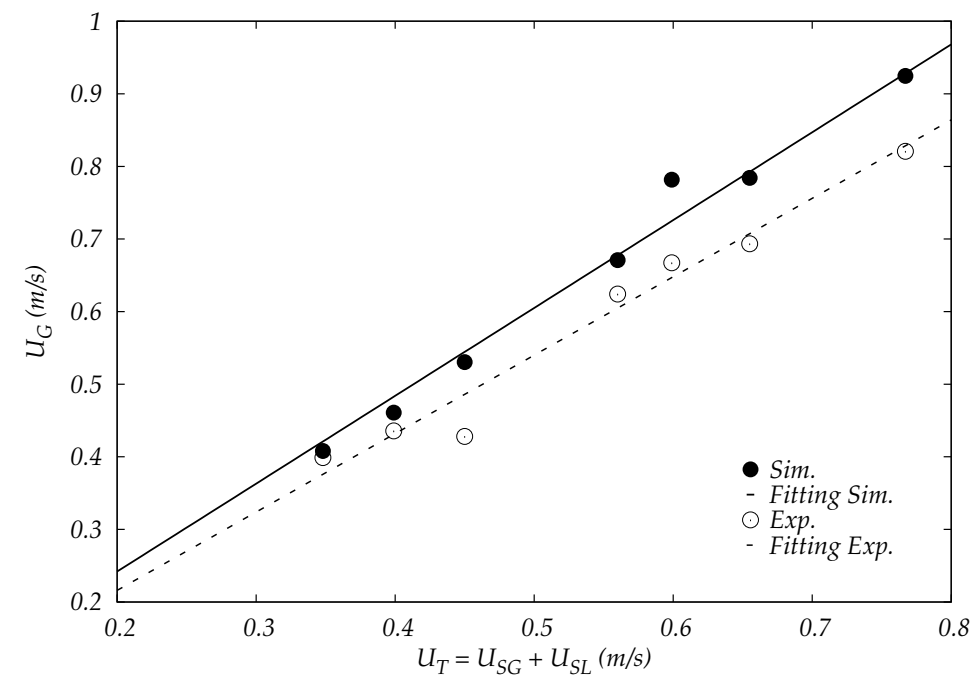

Figure 16: Bubble velocity as a function of the mixture superficial velocity. Symbols: experimental and numerical data. Lines: linear fittings of experimental and numerical data.

Rewriting Eq. 18, a prediction of the mean void fraction as a function of the ratio between the gas and liquid superficial velocities can be obtained:

$$
\alpha=\frac{1}{C_{0}}\left(1+\frac{1}{\frac{U_{S G}}{U_{S L}}}\right)^{-1}
$$

The mean void fraction was estimated from the bubble velocity and the gas superficial velocity with:

$$
\alpha=\frac{U_{S G}}{U_{G}}
$$

Figure 17 shows the mean void fraction as a function of the ratio between gas and liquid superficial velocities. The theoretical prediction given by Eq. 19 is plotted for both experimental and numerical simulations data by using their respective $C_{0}$. The behaviour of the void fraction obtained in the simulations reproduces the experimental behaviour and coincides with the theoretical prediction. In agreement with the slightly larger $U_{G}$ observed in simulations in comparison with experiments in Fig. 16, one can observe smaller void fraction values in the simulations than in the experiments in Fig. 17. 


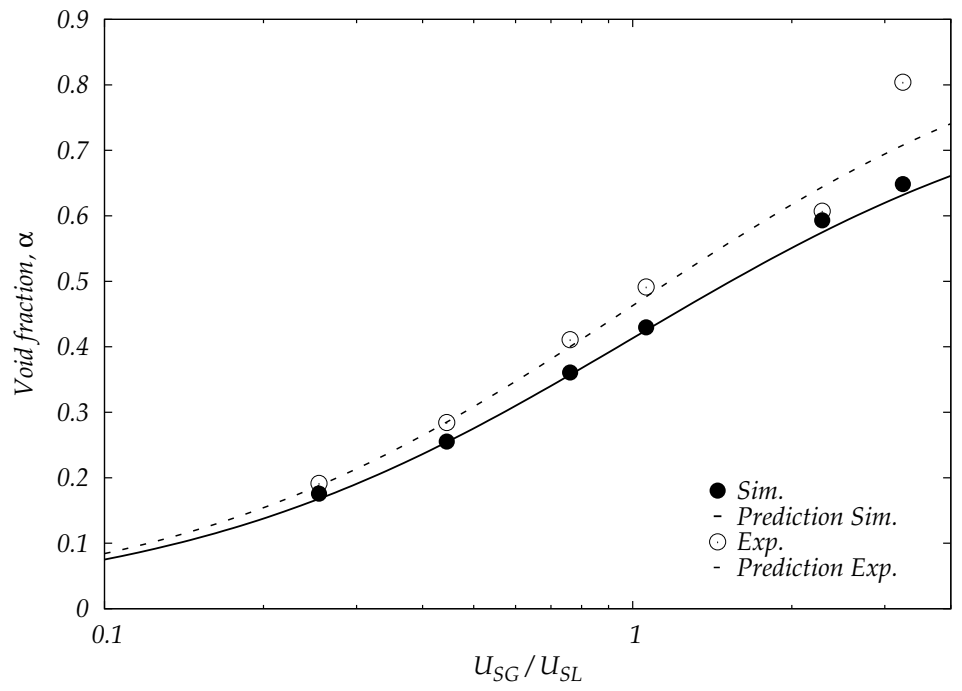

Figure 17: Void fraction as a function of the ratio between gas and liquid superficial velocities. Symbols: experimental and numerical data. Lines: theoretical prediction given by Eq. 19 .

\subsection{Bubble generation frequency}

In order to ensure regularity in the formation of bubbles, we generated trains of bubbles containing at least four of them. The generation frequency was estimated by measuring the time required to generate the bubbles. The first bubble of each train, which was strongly dependent on the initial geometry as previously remarked, was not considered.

Fig. 18 shows the bubble frequency as a function of the superficial gas velocity at $U_{S L}=0.106,0.318$ and $0.531 \mathrm{~m} / \mathrm{s}$ for both experimental and numerical simulation data. Lines correspond to the fitting of the experimental data [6]. It can be observed that the frequency increases with the superficial liquid velocity. Indeed, the drag due to the liquid cross-flow increases when the superficial liquid velocity is increased, thus causing the reduction of the gas thread and bubble size [5, 6].

Although the simulation data are qualitatively very similar to the experimental results, it can be observed that frequency values are always slightly larger in the simulations. This is associated to the observation of smaller bubbles in the simulations. This discrepancy could be explained by the fact that simulations were run in $2 \mathrm{D}$ and with liquid viscosities different than the experimental ones. However, there is no clear evidence of which of these two effects plays a more important role in this case.

The dimensionless frequency, or Strouhal number $S t=f \phi / U_{G}$, is represented 


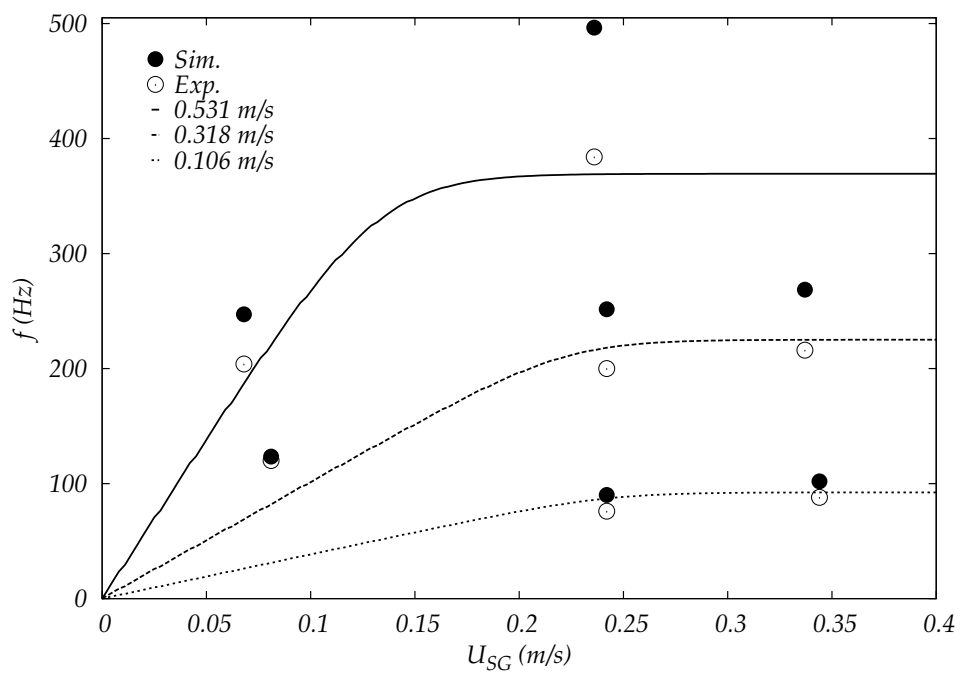

Figure 18: Bubble frequency as a function of the superficial gas velocity for different superficial liquid velocities. Symbols: experimental and simulation results. Lines: fitting of the experimental data [6].

in Fig. 19 as a function of the void fraction for both experiments and numerical simulations. Line represents the theoretical approximation for $S t$ in the saturation regime, given by [32]:

$$
S t=0.6\left(1-C_{0} \alpha\right)
$$

For $\alpha>0.2$ experimental and numerical data show a linear behaviour in accordance with Eq. 21. Points with $\alpha<0.2$ correspond to the linear regime and therefore we could not expect an agreement with Eq. 21. Once again, the similarity is noticeable between the experimental and simulation data despite the different geometry.

\subsection{Bubble and unit cell lengths}

The bubble length $L_{B}$ was directly measured from simulations and compared to the experimental data. Bubble length, considered as the gas displacement during the time required to generate one bubble $1 / f$, can be estimated from:

$$
L_{B}=\frac{U_{S G}}{f}
$$

Fig. 20 shows the dimensionless measured bubble length in simulations and experiments as a function of $U_{S G} / f \phi$, as well as the expected behaviour given by 


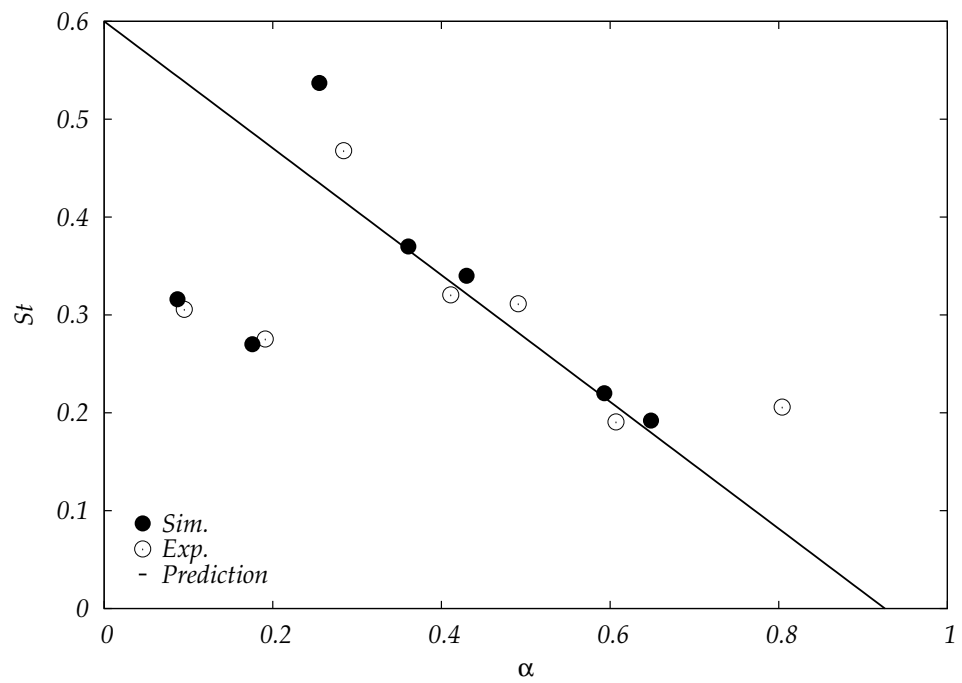

Figure 19: Strouhal number as a function of the void fraction. Symbols: experimental and simulation data. Line: theoretical approximation (Eq. 21).

Eq. 22. One can observe that bubble lengths were underpredicted by Eq. 22. This disagreement can be explained by the fact that Eq. 22 corresponds to the length that bubbles would have in case they filled the whole capillary cross-section and were not longitudinally deformed by the liquid drag. Fig. 21 shows the distinction between the bubble length given by Eq. 22 and the actual bubble length which was measured in the simulations and experiments. It can also be observed that the experimental bubble lengths are slightly greater than the ones in the $2 \mathrm{D}$ simulations, which agrees with the fact that the generation frequencies are smaller in the experiments.

According to previous analyses $[4,5,6,7]$, the bubble generation frequency is basically controlled by the gas and liquid flow rates, and hence must be $L_{B}$. Fig. 22 shows the dimensionless bubble length as a function of the liquid superficial velocity. An increase in bubble size can be observed when increasing $U_{S G}$, as well as a decrease in bubbles size is observed when $U_{S L}$ is increased. In addition, a linear relationship between $L_{B}$ and the gas and liquid superficial velocities ratio can be observed in Fig. 23, where the same slope of $\sim 1.14$ is obtained in the experiments and in the numerical simulations. The behaviours shown in Figs. 22 and 23 is in agreement with recently reported results [7, 42].

A high regularity in the generation of bubbles and in the bubble size was observed in the simulations. The standard deviation obtained was smaller than 0.1 , 


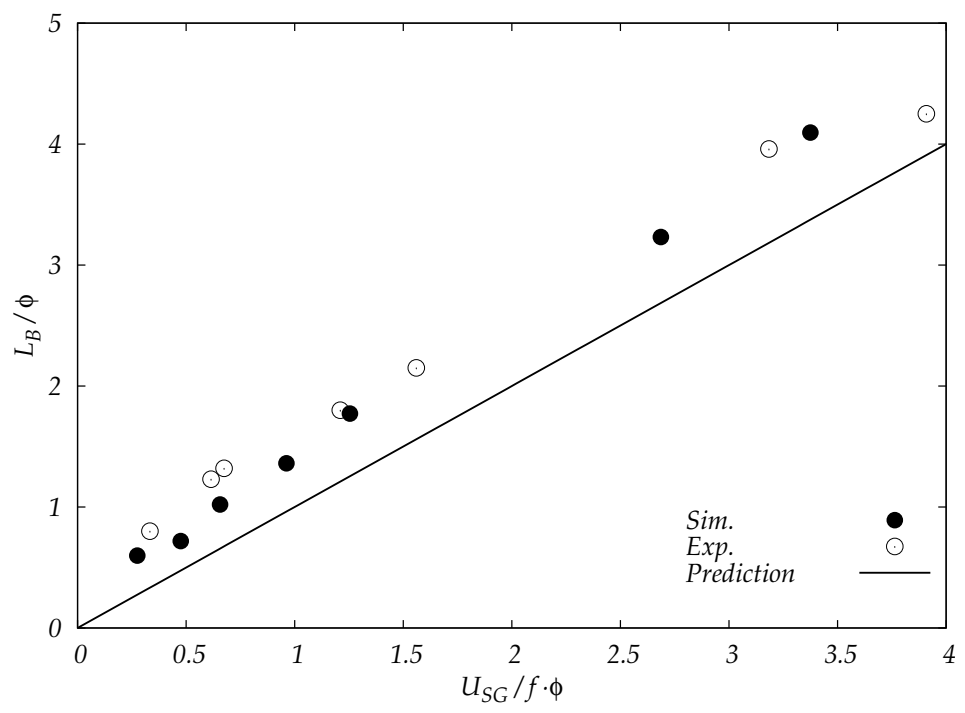

Figure 20: Dimensionless bubble length as a function of $U_{S G} / f \phi$. Symbols: experimental and numerical data. Line: theoretical prediction given by Eq. 22.
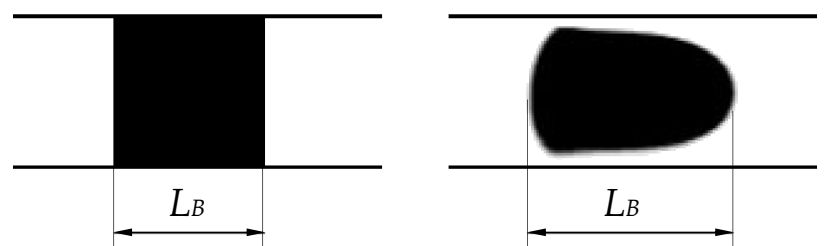

Figure 21: (Left) bubble length given by Eq. 22, and (right) actual bubble length. 


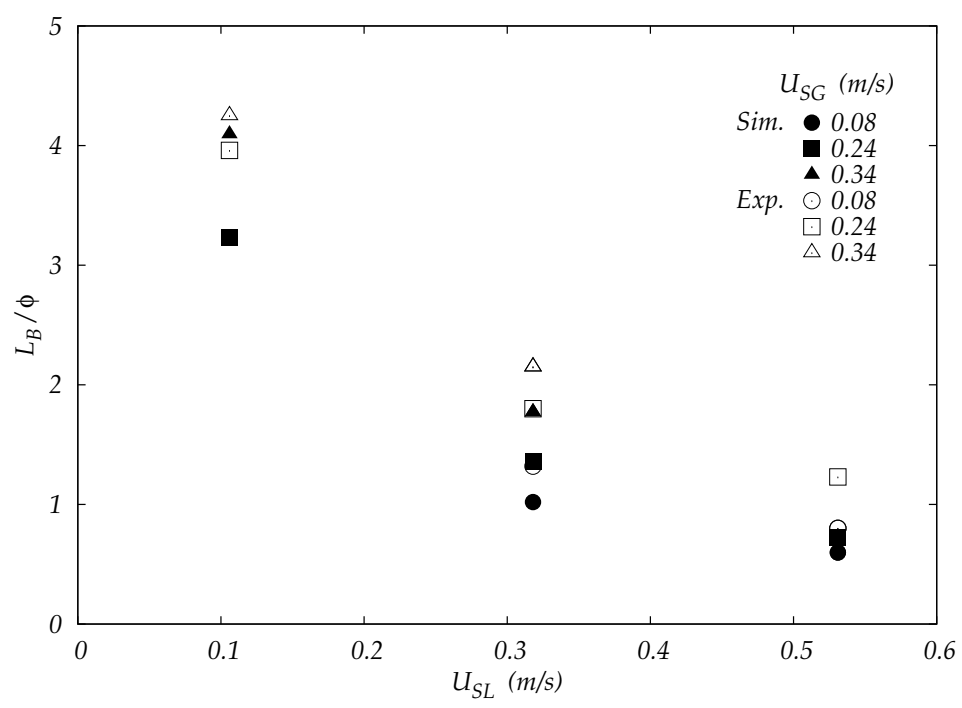

Figure 22: Dimensionless bubble length as a function of the liquid superficial velocity. Symbols: experimental and numerical data.

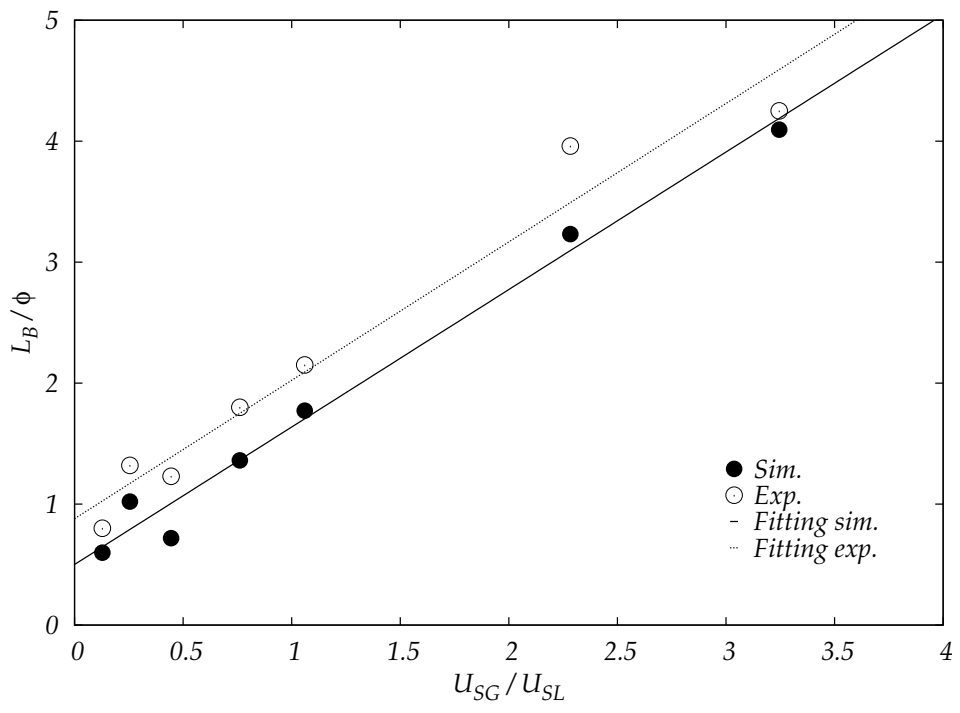

Figure 23: Dimensionless bubble length as a function of the gas and liquid superficial velocities ratio. Symbols: experimental and numerical data. Lines: fittings. 


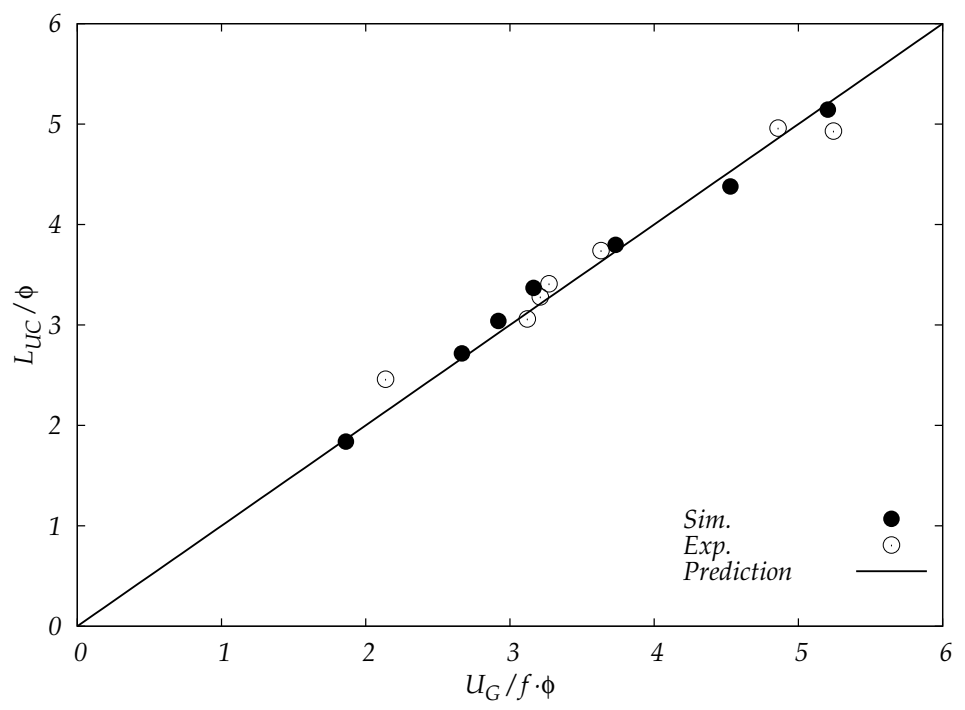

Figure 24: Dimensionless unit cell length as a function of $U_{G} / f \phi$. Symbols: experimental and numerical data. Line: theoretical prediction given by Eq. 23.

being this value two orders of magnitude smaller than the mean bubble length. Therefore, we assumed that each train of bubbles could be represented by a unit cell composed of one bubble and the liquid between two bubbles. The unit cell length $L_{U C}$ was calculated as the distance between the tip of two consecutive bubbles. Considering that $1 / f$ is the time required for a unit cell moving at a velocity $U_{G}$ to move a distance $L_{U C}$, the unit cell length can be expressed by:

$$
L_{U C}=\frac{U_{G}}{f}
$$

Fig. 24 shows the dimensionless measured unit cell length in simulations and experiments as a function of $U_{G} / f \phi$ and the expected behaviour given by Eq. 23 . In this case, both experiments and simulations coincide with the prediction.

\section{Conclusions}

We have presented a study of the formation of minibubbles in a T-junction by means of the fluid dynamics numerical code JADIM. Numerical simulation results were compared with previous experimental works.

A dimensional analysis based on the Buckingham's $\pi$ theorem was carried out in order to determine the dimensionless numbers controlling the generation and 
detachment of bubbles in the system. Simulations were carried out with the values of the gas and liquid superficial velocities used in the experiments. Only viscosity had to be changed in simulations from the experimental values in order to avoid the development of numerical spurious currents.

The numerical modelling of the T-junction required an accurate selection of boundary conditions and, in particular, of the contact angle between the gas-liquid interface and the walls of the system. Considering that we aimed to reproduce by means of numerical simulations in 2D the existing 3D experimental results, we focussed on the squeezing process of the gas thread in the T-junction. Since forces acting on the gas thread depend on the dimensionality, an artificial squeezing mechanism based on the observation of the thread behaviour from the experimental images was implemented in the numerical code in order to get rid of 2D effects.

The numerical generation of a train of bubbles in a mini-channel in conditions relevant to microgravity was satisfactorily obtained. In particular, numerical simulations reproduced the bubble and slug flow patterns observed experimentally. We obtained results on the behaviour of bubble velocity, void fraction, bubble generation frequency and bubble and unit cell lengths. A stable periodicity during the bubble generation and regularity in bubble size were observed. A linear and a saturation regimes in the bubble frequency as observed in the experiments were reproduced. Bubble shape and bubble position in the capillary centreline agreed with the behaviour observed in experiments. Bubble length and unit cell length were also compared with experimental data.

Thus, since 2D simulations and 3D experimental results were very similar, we can conclude that the squeezing process and, in particular, the orientation of the gas thread in time, fully determine the characteristics of the generated flows. In fact, we have shown that $2 \mathrm{D}$ numerical simulations can reproduce $3 \mathrm{D}$ flow characteristics in other regimes of the T-junction, provided that an adequate squeezing mechanism is implemented. In addition, when no artificial squeezing mechanism is applied in our simulations, one would expect to generate flows similar to those which would be observed in $2 \mathrm{D}$ or quasi-2D experiments.

We conclude that JADIM is an appropriate tool for the numerical study of twophase flows generated in a T-junction. Future works will focus on 3D simulations to make possible a direct comparison with experiments, specially concerning the pinch-off mechanism. This may allow a future exploration of parameter regimes of the system which are difficult to achieve experimentally. 


\section{Acknowledgements}

This work has been financially supported by the Spanish Ministerio de Ciencia e Innovación (Project AYA2009-11493). S.A acknowledges the financial support from the Spanish Ministerio de Educación (Movility Grant for Doctoral Students). S.A. is indebted to the Institute de Mécanique des Fluides de Toulouse for its kind hospitality and assistance. R.G.C. is indebted to the Center for System Studies at the University of Alabama in Huntsville for its kind hospitality during his sabbatical leave.

\section{References}

[1] Ostrach S. Industrial processes influenced by gravity. NASA CR-182140, C21066-G, 1988.

[2] McQuillen J., Colin C., and Fabre J. Ground-based gas-liquid flow research in microgravity conditions: state of knowledge. Space Forums 3, 1998: 165203.

[3] Ohta H., Baba A., and Gabriel K. Review of existing research on microgravity boiling and two-phase flow. Ann. N.Y. Acad. Sci., 2002; 974: 41.

[4] Carrera J., Ruiz X., Ramírez-Piscina L., Casademunt J., and Dreyer M. Generation of a monodisperse microbubble jet in microgravity. AIAA Journal, 2008: 46(8): 2010-2019.

[5] Arias S., Ruiz X., Ramírez-Piscina L., Casademunt J., and González-Cinca R. Experimental study of a microchannel bubble injector for microgravity applications. Microgravity Sci. Technol, 2009; 21: 107-111.

[6] Arias S., González-Cinca R., Ruiz X., Ramírez-Piscina L., and Casademunt J. Characterization of the performance of a minibubble generator in conditions relevant to microgravity. Colloids and Surfaces A: Physicochem. Eng. Aspects, 2010; 365: 52-55.

[7] Fu T., Ma Y., Funfschilling D., Zhu C., and Li. H.Z. Squeezing-to-dripping transition for bubble formation in a microfluidic T-junction. Chem. Eng. Sci., 2010; 65(12): 3739-3748.

[8] Qian D., and Lawal A. Numerical study on gas and liquid slugs for Taylor flow in a T-junction microchannel. Chem. Eng. Sci., 2006; 61: 7609-7625. 
[9] Kashid M.N., Platte F., Agar D.W., and Turek S. Computational modelling of slug flow in a capillary microreactor. J. Comput. Appl. Math., 2007; 203: 487-497.

[10] Menech M. De, Garstecki P., Jousse F., and Stone H.A. Transition from squeezing to dripping in a microfluidic T-shaped junction. J. Fluid Mech., 2008; 595: 141-161.

[11] Magnaudet J., Rivero M., and Fabre J. Accelerated flows past a rigid sphere or a spherical bubble. Part 1. Steady straining flow. J. Fluid Mech., 1995; $97-$ 135.

[12] Legendre D., and Magnaudet J. The lift force on a spherical bubble in a viscous linear shear flow. J. Fluid. Mech., 1998; 368: 81-126.

[13] Legendre D., Magnaudet J., and Mougin G. Hydrodynamic interactions between two spherical bubbles rising side by side in a viscous liquid. J. Fluid Mech., 2003; 497: 133-166.

[14] Legendre D. On the relation between the drag and the vorticity produced on a clean bubble. Phys. Fluids, 2007; 19: 018102.

[15] Legendre D., and Colin C. Enhancement of wall-friction by fixed capbubbles. Phys. Fluids, 2008; 20: 051704.

[16] Legendre D., Lauga E., and Magnaudet J. Influence of slip on the dynamics of two-dimensional wakes. J. Fluid. Mech., 2009; 633: 437-447.

[17] Bonometti T., and Magnaudet J. Transition from spherical cap to toroidal bubbles. Phys. Fluids, 2006; 18: 052102.

[18] Bonometti T., and Magnaudet J. An interface capturing method for incompressible two-phase flows. Validation and application to bubble dynamics. Int. J. of Multiphase Flow, 2007; 33(2): 109-133.

[19] Sarrazin F., Bonometti T., Prat L., Gourdon C., and Magnaudet J. Hydrodynamic structures of droplets engineered in rectangular micro-channels. Micro. Nano, 2008; 5: 131-137.

[20] Dupont J.-B., and Legendre D. Numerical simulation of static and sliding drop with contact angle hysteresis. J. Comput. Phys., 2010; 229: 2453-2478. 
[21] Brackbill J.U., Kothe D.B., and C. Zemach. A continuum method for modelling surface tension. Journal of Computational Physics, 1992; 100(2): 335354.

[22] Scardovelli R., and Zaleski S. Direct numerical simulation of free surface and interfacial flow. Ann. Rev. Fluid Mech., 1999; 31: 567.

[23] Sethian J. Level Set methods and fast marching methods. Cambridge University Press, Cambridge, 1999.

[24] Sussman M., Fatemi E., Smereka P., and Osher S. A Level Set approach for computing solutions in incompressible two-phase flows. Comput. Fluids, 1998; 27: 567.

[25] Zalesak S.T. Fully multidimentional flux-corrected transport algorithms for fluids. J. Comput. Phys., 1979; 31: 335-362.

[26] Lafaurie B., Nardone C., Scardovelli R., Zaleski S., and Zanetti G. Modelling merging and fragmentation in multiphase flows with SURFER. J.Comp.Phys., 1994; 113: 134-147.

[27] Popinet S., and Zaleski S. A front-tracking algorithm for accurate representation of surface tension. Int. J. Numeric. Meth. Fluid, 1999; 30: 775-793.

[28] Ménétrier-Deremble L., and P. Tabeling. Droplet breakup in microfluidic junctions of arbitrary angles. Phys. Rev. E, 2006; 74: 035303(R).

[29] SteijnV. van, Kleijn C.R., and Kreutze M.T. Flows around Confined Bubbles and Their Importance in Triggering Pinch-Off. Physical Review Letters, 2009; 103: 214501.

[30] Dukler A.E., Fabre J.A., McQuillen J.B., and Vernon R. Gas-liquid flow at microgravity conditions: flow patterns and their transitions. International Journal of Multiphase Flow, 1998; 14: 389-400.

[31] Suo M., and Griffith P. Two-phase flow in capillary tubes. J. Basic Eng., 1664; 86: 576-582.

[32] Arias S., and González-Cinca R. Introduction of the Strouhal number in the analysis of the bubble generation frequency in minichannels in microgravity related conditions. (In preparation). 
[33] Nicklin D.J., Wilkes J.O., and Davidson J.F. Two phase flow in vertical tubes. Trans. Inst. Chem. Eng., 1962; 40: 61-68.

[34] Colin C, Fabre J., and McQuillen J. Bubble and slug flow at microgravity conditions: state of knowledge and open questions. Chem. Eng. Comm., 1996; 141/142: 155-173.

[35] Bousman W.S., McQuillen J.B., and Witte L.C. Gas-liquid flow patterns in microgravity: effects of tube diameter, liquid viscosity and surface tension. Int. J. Multiphase Flow, 1996; 22(6): 1035-1053.

[36] Taylor G.I. Deposition of a viscous fluid on the wall of a tube. J. Fluid Mech., 1961; 10(2): 161-165.

[37] Bretherton F.P. The motion of long bubbles in tubes. J. Fluid Mech., 1961; 10: $166-188$.

[38] Aussillous P., and Quéré D. Quick deposition of a fluid on the wall of a tube. Phys. Fluids, 2000; 12: 2367-2371.

[39] Turnau J.-R., Ha Ngoc H., and Fabre J. The motion of long bubbles in tube at negligible gravity. 6th International Conference on Multiphase Flow, ICMF 2007, Leipzig, Germany.

[40] Ha Ngoc H., and Fabre J. The velocity and shape of 2D long bubbles in inclined channels or in vertical tubes. Part I: in a stagnant liquid. Multiphase Sciences and Technology, 2004; 16: 175-188.

[41] Figueroa B., and Fabre J. Taylor bubble moving in a flowing liquid in vertical channel: transition from symmetric to asymmetric shape. J. Fluid Mech. (Accepted for publication).

[42] Garstecki P., Fuerstman M.J., Stone H.A., and Whitesides G.M. Formation of droplets and bubbles in a microfluidic T-junction-scaling and mechanism of break-up. Lab on a Chip, 2006; 6: 437-446. 